\title{
Photoelectron microscopy of organic surfaces: The effect of substrate reflectivity
}

\author{
Rudy J. Dam and O. Hayes Griffith \\ Institute of Molecular Biology and Department of Chemistry, University of Oregon, Eugene, Oregon, 97403 \\ Gertrude F. Rempfer \\ Department of Physics, Portland State University, Portland, Oregon 97207 \\ (Received 26 September 1975) \\ Photoelectron measurements of thin organic films deposited on a metal substrate may contain information \\ from deep within the sample, derived from reflected ultraviolet light. This effect depends on the reflectivity \\ of the substrate, the sample thickness and optical absorption coefficient, and the photoelectron escape \\ depth. Calculations are given for phthalocyanine as a specific example. Contrast reversal and apparent see- \\ through effects resulting from reflection are predicted in overlapping thin films. Photoelectron micrographs \\ of thin films and grid patterns of phthalocyanine show that the reflection model is essentially correct. This \\ effect can be substantially reduced by using a nitrocellulose-coated carbon substrate.
}

PACS numbers: 07.80., 87.20., 79.60., 81.50.Q

\section{INTRODUCTION}

Recently a number of laboratories have begun to investigate organic and biological materials by surface physics techniques such as ultraviolet and $\mathrm{x}$-ray photoelectron spectroscopy ${ }^{1-5}$ and photoelectron microscopy ${ }^{6-10}$ In these experiments, the organic sample is placed on a metal surface, whereas in the classical experiments the metal surface itself is the sample. During the photoelectric experiments novel effects sometimes arise that may be caused by the metal substrate. An example from photoelectron microscopy is shown in Fig. 1. These micrographs of overlapping phthalocyanine layers, deposited on stainless steel, are formed by electrons photoejected from the sample, accelerated, and focused by electron optics to produce an image of the surface. They show two interesting features. Both micrographs give the appearance of seeing through the top layer, clearly delineating the regions of overlap. Second, the sense of contrast depends on the film thicknesses. In Fig. 1(a), the region of overlap, $400 \AA$ thick, appears dark compared to the adjacent layers, 150 and $250 \AA$ thick. In Fig. $1(\mathrm{~b})$, the region of overlap $(150 \AA)$ appears bright compared to the adjacent layers (50 and $100 \AA$ ). Similar photoelectron micrographs, using considerably thicker overlapping sections of methacrylate, have been observed by Engel ${ }^{8}{ }^{8}$ In this paper we propose a mechanism for the observed thickness dependence, based on the effect of ultraviolet light reflected from the substrate, and suggest substrates for studying organic and biological surfaces that minimize this effect.

\section{MATERIALS AND METHODS}

The photoelectron microscope used in this experiment is an ultrahigh vacuum instrument described previously. ${ }^{6}$ In this instrument the sample is illuminated at nor mal incidence with ultraviolet light. Sample rods of polished stainless steel, $6.35 \mathrm{~mm}$ in diameter, were used in studying the effect of a reflecting substrate. Nonreflecting substrates were prepared by depositing a layer of carbon on one end of the rods by vacuum evaporation. Carbon film thicknesses were determined by optical absorption at $400 \mathrm{~nm}$ of a film si- multaneously evaporated onto a glass microscope cover slip. The absorption coefficient of Cosslett and Cosslett ${ }^{11}$ was used for these measurements. To suppress photoemission from the carbon coating, a thin film of nitrocellulose was deposited over the carbon. The carboncoated sample rods were placed in a Büchner funnel equipped with drainage hose and clamp and filled with enough distilled water to cover the rod end surface to a depth of approximately $1 \mathrm{~cm}$. One drop of $1 \%$ nitrocellulose in amyl acetate was placed on the water surface and the solvent allowed to evaporate. The water was slowly removed from the bottom of the funnel, allowing the nitrocellulose film to settle onto the end of the sample rod. The rods were air dried at room temperature. Nitrocellulose was carefully removed from the sides of the rod by wiping with a cotton swab dipped in acetone. Metal-free phthalocyanine (Eastman Kodak Co., Rochester, N.Y.) was purified, and thin films were vacuum evaporated as described previously. ${ }^{12,13}$ The grid patterns were formed by evaporating through a standard 200-mesh electron microscope copper grid and then
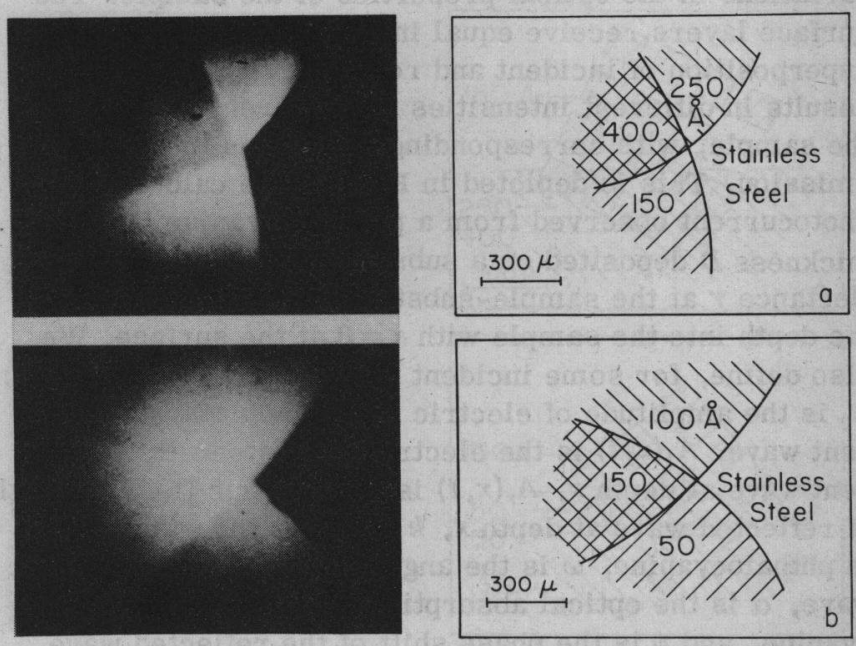

FIG. 1. (a) Photoelectron micrograph of overlapping layers of phthalocyanine on stainless-steel substrate. Film thicknesses were $150 \AA$ followed by $250 \AA$. (b) Overlapping layers of phthalocyanine on stainless steel, $100 \AA$ followed by $50 \AA$. 


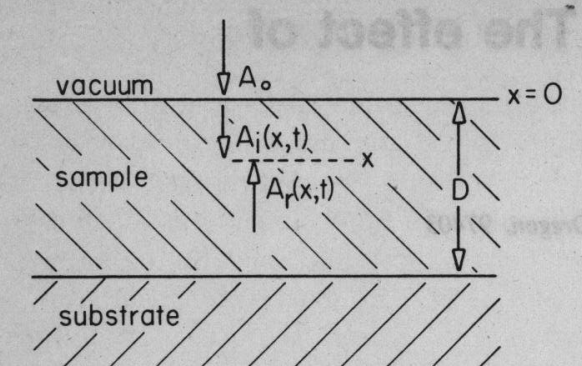

FIG. 2. Calculation of the reflection effect. Ultraviolet light
of amplitude $A_{0}$ strikes a sample of thickness $D$ at normal incidence. After penetrating the sample a fraction $r$ of the wave
is reflected from the metal substrate. At an arbitrary is reflected from the metal substrate. At an arbitrary depth $x$ the resultant wave motion $A(x, t)$ is calculated as the sum
of an incident component $A_{i}(x, t)$ and a reflected component
$A_{\gamma}(x, t)$. removing the grid. Film thicknesses for phthalocyanine
were also determined by optical absorption at $400 \mathrm{~nm}$, using an absorption coefficient of $0.64 \times 10^{5} \mathrm{~cm}^{-1}$ as reported by Schechtman. ${ }^{13}$ The thickness measurements were verified with a Varian $\AA$-scope interferometer. Phythalocyanine film thicknesses reported in this pape are believed accurate to $\pm 20 \%$. For all photoelectron micrographs presented here, the wavelength of the incident light was $220 \pm 10 \mathrm{~nm}$ and the exposure time was $8 \mathrm{sec}$. The micrographs were recorded from the image intensifier on Kodak Tri-X emulsion and developed for $3(\mathrm{~m})$ in full strength Kodak Dektol developer at $68^{\circ} \mathrm{F}$ ture in the photoelectron microscope

\section{CALCULATION OF THE REFLECTIVITY EFFECT}

The following model is based on normally incident light penetrating an optically thin sample, reflecting from the substrate, and returning to the sample surface. is neglected. Light reflected from the substrate carry information from deep within the sample (rela to the photoelectron escape depth) corresponding to variations in the optical properties of the sample. surface layers receive equal incident light, but the superposition of incident and reflected light, in genera results in different intensities for different areas of the sample, with corresponding variations in photoemission. This is depicted in Fig. 2. We calculate the photocurrent observed from a phythalocyanine film of thickness $D$ deposited on a substrate with amplitude reflectance $r$ at the sample-substrate interface. Let $x$ be the defin $A_{0}$ is the amplitude of electric field component of incident wave, $A_{1}(x, t)$ is the electric field strength of incident wave at depth $x, A_{r}(x, t)$ is the electric field strength of reflected wave at depth $x, k \equiv 2 \pi / \lambda$ is the wavenumber in phthalocyanine, $\omega$ is the angular frequency of the wave, $\alpha$ is the optical absorption coefficient for phthalocyanine, and $\psi$ is the phase shift of the reflected wave. The incident and once reflected waves at depth $x$ are represented by the real parts of

$A_{i}(x, t)=A_{0} \exp \left(-\frac{1}{2} \alpha x\right) \exp [i(\omega t-k x)]$

362 J. Appl. Phys., Vol. 47, No. 3, March 1976
$A_{r}(x, t)=r A_{0} \exp \left[-\frac{1}{2} \alpha(2 D-x)\right] \exp \{i[\omega t-k(2 D-x)+\psi]\}$. The resultant wave motion is the superposition of the real part of

$A(x, t)=A_{0} \exp (i \omega t)\left\{\exp \left[-\left(\frac{1}{2} \alpha+i k\right) x\right]\right.$ $\left.+r \exp \left[-\left(\frac{1}{2} \alpha+i k\right)(2 D-x)+i \psi\right]\right\}$.

The intensity $I(x)$ is proportional to $A A^{*}$ :

$I(x)=I_{0}\left\{\exp (-\alpha x)+\gamma^{2} \exp [-\alpha(2 D-x)]\right.$

$+2 r \exp (-\alpha D) \cos [2 k(D-x)-\psi]\}$

where $I_{0} \propto A_{0}^{2}$. Defining $N(x) d x$ to be the photocurent contribution from $d x$ at depth $x$, the

$N(x) d x \propto I(x) \exp (-x / \sigma) d x$

(s) $\sigma$ is a characteristic (energy-averaged) photoelectron escape depth. Combining Eqs. (2) and (3) and integrating through the sample depth yields the total photoelectron current $N$ 。 The result is

$N \propto 2 \gamma I_{0} \exp (-\alpha D)\left\{\left(1 / \sigma^{2}-\alpha^{2}\right)^{-1 / 2}\left[\cosh \left(\alpha D-\ln \gamma-\psi_{\alpha}\right)\right.\right.$

$\left.-\exp (-D / \sigma) \cosh \left(\ln \gamma+\psi_{\alpha}\right)\right]$

$+\left(1 / \sigma^{2}+4 k^{2}\right)^{-1 / 2}\left[\cos \left(2 k D-\psi-\psi_{k}\right)\right.$

$\left.-\exp (-D / \sigma) \cos \left(\psi+\psi_{k}\right)\right]$

The new quantities introduced in this equation are $\psi_{c}$ $=\operatorname{arctanh}(\alpha \sigma)$ and $\psi_{k}=\arctan (2 k \sigma)$. The terms multiplied by $\left(1 / \sigma^{2}-\alpha^{2}\right)^{-1 / 2}$ correspond to the photocurrent which would be predicted if interference of the inciden and reflected waves were ignored. The terms multi-

For metal-free phthalocyanine (Pc), $\alpha$ at $220 \mathrm{~nm}$ is $2.7 \times 10^{-3} \AA^{-1}, 13 k=2 \pi / \lambda$ (in Pc) $=2 \pi / 1350 \AA$, and $\sigma$ is $10-15 \AA .12$ Thus, $1 / \sigma \gg \alpha, 1 / \sigma \gg k$, and with these simplifying approximations Eq. (4) reduces to

$N \propto \sigma I_{0}\left[\left[1+\gamma^{2} \exp (-2 \alpha D)-\left(1+\gamma^{2}\right) \exp (-\alpha D) \exp (-D / \sigma)\right]\right.$

$+2 r \exp (-\alpha D)[\cos (2 k D-\psi)-\exp (-D / \sigma) \cos \psi]\}$.

The part in the first square brackets represents the current arising from the sum of separate intensities of incident and reflected waves, while the second part incorporates the interference factors. In Fig. 3, the photocurrent function of Eq. (5) is plotted versus $D$ for

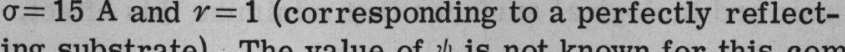
bination of dent light. For the curve in Fig. 3, the value $\psi=135^{\circ}$ was used as an estimate based on "the optical propertie of typical metals. The curve predicts a photoemission maximum in the region of $200 \AA$ Asmple thickness. This may be understood physically as follows. Consider a given value of $\sigma$. At thicknesses less than $\sigma$, photoemission will increase steadily with $D$. Essentially all photoelectrons produced in the sample are collected. As $D$ approaches $\sigma$, the photocurrent begins to level of more photoelectrons are being produced, but those pro-

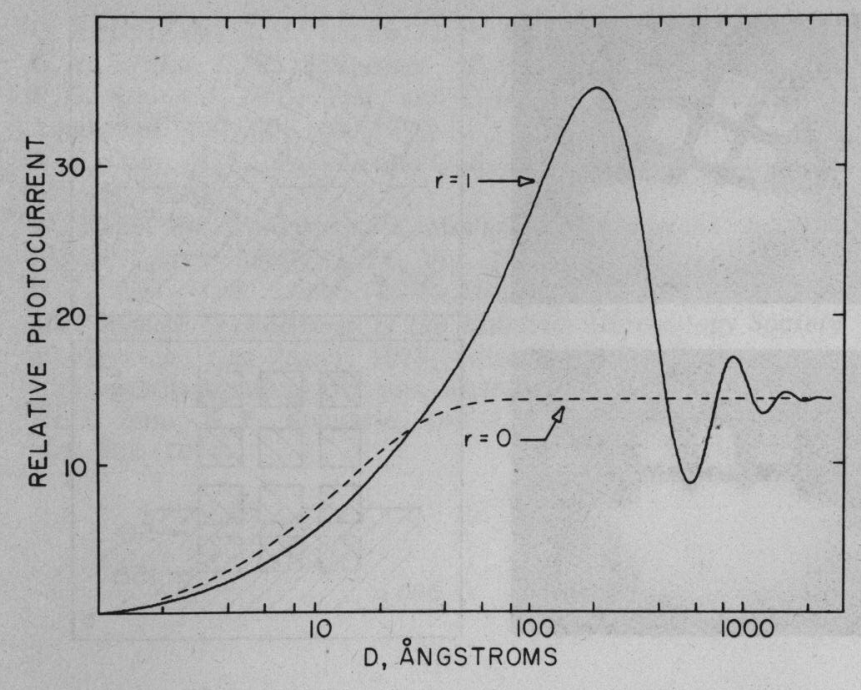

FIG. 3. Relative photocurrent $(N)$ as a function of sample these calculations were $\alpha=0.0027 \AA^{-1}, \sigma=15 \AA, k=0.0047$ $\AA^{-1}$, and $\psi=3 \pi \pi$. Solid curve: perfectly reflecting substrate
$(=1)$. Dashed curve: perfectly transmitting or absorbing
substrate $(r=0)$.

face and are therefore not collected. If there were no reflected light, the photocurrent would reach a constan value asy mptotically with $D$. This is shown by the dashed curve of Fig. 3, obtained by setting $r=0$ in Eq. (5). For a reflecting substrate, the reflected light wave combines coherently with the incident wave. The uv intensity profile has maxima approximately at the antinodes the incinter and tions of the maxima depend on the wavelenth of the light in the medium and also on the phase shift $y$ occurring on reflection. When maximum observed photoemissio occurs, there is a uv intensity maximum near the sample surface. Contrast reversals can arise from changes in either wavelength or sample thickness.

The reflection effect can strongly alter the observed photoemission, enhancing it by a factor of as much as 4 or reducing it to nearly zero. The effects are most prominent when the reflection coefficient is near unity and the absorption coefficient $\alpha$ is small compared with the wave number $k$. The curve for phthalocyanine in Fig. 3 shows that the photocurrent at maximum is greater by a factor of 2.5 than that for a nonreflecting substrate. It is notable that for small values of $D$ photoemission is actually predicted to decrease relative to that from a sample on an absorbing substrate. This is due to destructive interference between the incident and an be as arge as an order of masnitule at some sor ple depths. As $D$ becom appreciably la sor than $\alpha^{-1}$, optical absorption greatly reduces the reflected light component near the sample surface. Even though photoelectrons may be produced in deeper regions, only those within a few $\sigma$ from the surface are collected, and the photoemission curve for large $D$ approaches that predicted for an absorbing substrate.

863 J. Appl. Phys., Vol. 47, No. 3, March 1976
EXPERIMENTAL RESULTS AND DISCUSSION

Two sample geometries, uniform films and grid patterns of phthalocyanine, were examined. Typical results are shown in Figs. 1 and 4, respectively. The photoelectron micrographs of Fig. 1 are in qualitative agreement with the reflection calculations for the known
value of $\sigma, 15 \AA$. In Fig. 1 (a), the overlapping phthalovalue of $\sigma, 15 \AA$. In Fig. $1(\mathrm{a})$, the overlapping phthalo-
cyanine film thicknesses are greater than $D_{\max }$. Thicker regions are thus predicted to photoemit less than thinregions are thus predicted to photoemit less the the
ner regions. This is the observed result, since the overlap region appears darker in Fig. 1(a). In Fig. 1(b), the overlapping film thickness is less than $D_{\max }$. In this case, the thicker overlap region appears brighter than the two thin films, in agreement with the calculations. The reflection effect is also evident in the grid patterns of Fig. 4. The film thicknesses are identical to those of Fig. 1, but in this case square patches of phthalocyanine were prepared first and then partially covered with uniform layers of phthalocyanine. The uncovered square patches of phthalocyanine are bright compared lower half of Fig, s( substrate. The grid pattern in the nine has reversed contrast. In Fig. $4(\mathrm{~b})$, there is no
nof contrast reversal but there is an illusion of seeing through the $50-\AA$ overlayer. These effects are all predicted by the above calculations and are consistent with the uniform film data of Fig. 1 .

While the above calculations were derived for partialabsorbing organic or biological thin films, they are also relevant to several observations on inorganic samples. Möllenstedt, Speidel, and $\mathrm{Koch}^{14}$ observed intererence fringes in photoelectron images of thin metal and carbon films separated from a reflective substrate wedge-shaped transparent dielectric support. These dielectric. Wegmann ${ }^{15}$ reported interference effects in photoelectron micrographs of thin semiconductor films; he ascribed them to standing waves combined with a pho-
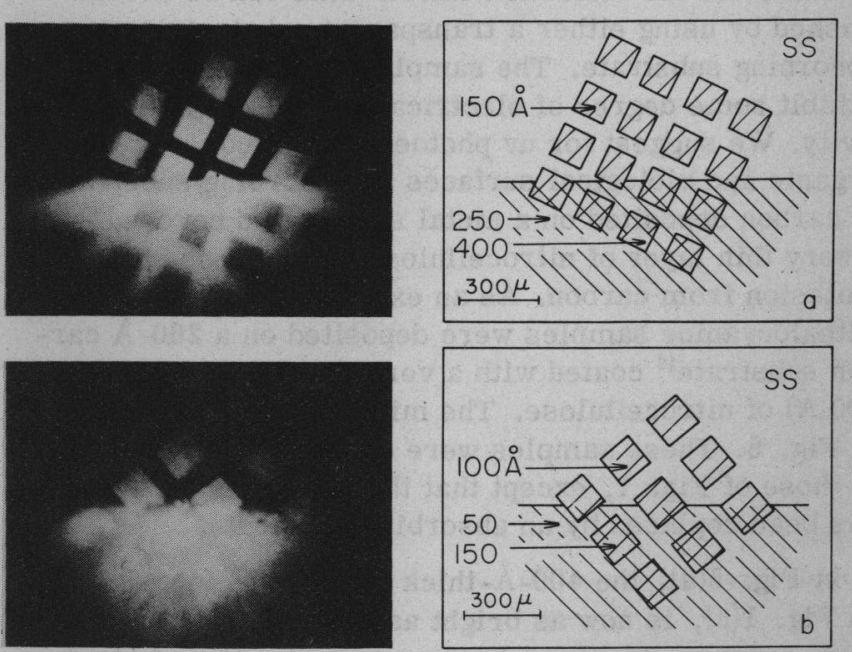

FIG. 4. (a) Phthalocyanine grid pattern, $150 \AA$ thick, partially covered with an additional $250 \AA$. Stainless-steel substrate.
(b) Phthalocyanine grid pattern, $100 \AA$ thick, partially covered (b) Phthalocyanine grid pattern, $100 \AA$ thick, partially
with an additional $50 \AA$. Stainless-steel substrate. 

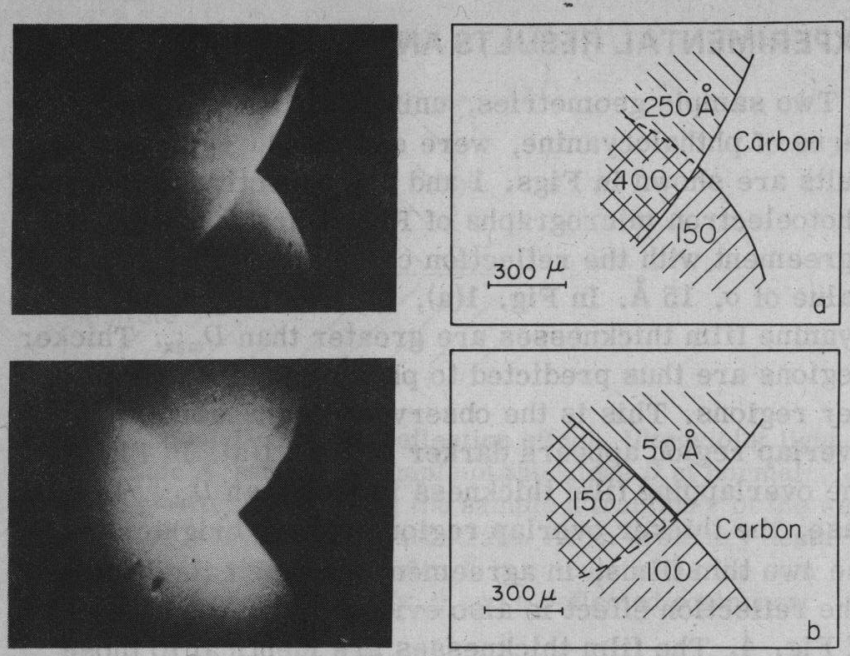

FIG. 5. (a) Overlapping layers of phthalocyanine on carbon$250 \AA$. (b) Overlapping layers of phthalocyanine on carbonnitrocellulose substrate. Film thicknesses $100 \AA$ followed

toelectron escape depth which is small compared with the wavelenth of trate rection to to apping sections of methyl methacrylate reported by Engel. ${ }^{8}$

Besides photoelectron microscopy, the substrate relection effect can alter uv photoelectron spectroscopy tron spectroscopy data. It has not to our knowledge be aken into account in the photoelectron spectroscopy literature. However, it could be significant in quantita tive measurements, particularly since substrate reflectivity varies with wavelength of incident light. In all of these experimental approaches it is desirable to be able to minimize the reflection effect. This can be accomplished by using either a transparent substrate or an absorbing substrate. The sample substrate must also exhibit some degree of electrical and thermal conductivity. We suggest for uv photoelectric studies of of very thin layer of nitrocellu lose to suppress photoemission from carbon. As an example a series of phthalocyanine samples were deposited on a $260-\AA$ carbon substrate ${ }^{16}$ coated with a very thin film (e. g., $20-$ $00 \AA$ A) of nitrocellulose. The micrographs are shown in Fig. 5. These samples were essentially identical to those of Fig. 1, except that the reflecting substrate has been replaced by an absorbing substrate.

In Fig. 5(a), the $400-\AA$-thick overlap region, dark in Fig. 1(a), is now as bright as the $250-\AA$ film. In Fig。. $5(b)$, some contrast can be seen between 50 and $150 \AA$ as predicted (see dashed line in Fig. 3), but the boundary between 150 and $100 \AA$ is no longer visible as it was in Fig. 1(b). Another way of showing the absence of reflec-
tion effects is illustrated in Fig. 6, in which grid pat-

864 J. Appl. Phys., Vol. 47, No. 3, March 1976
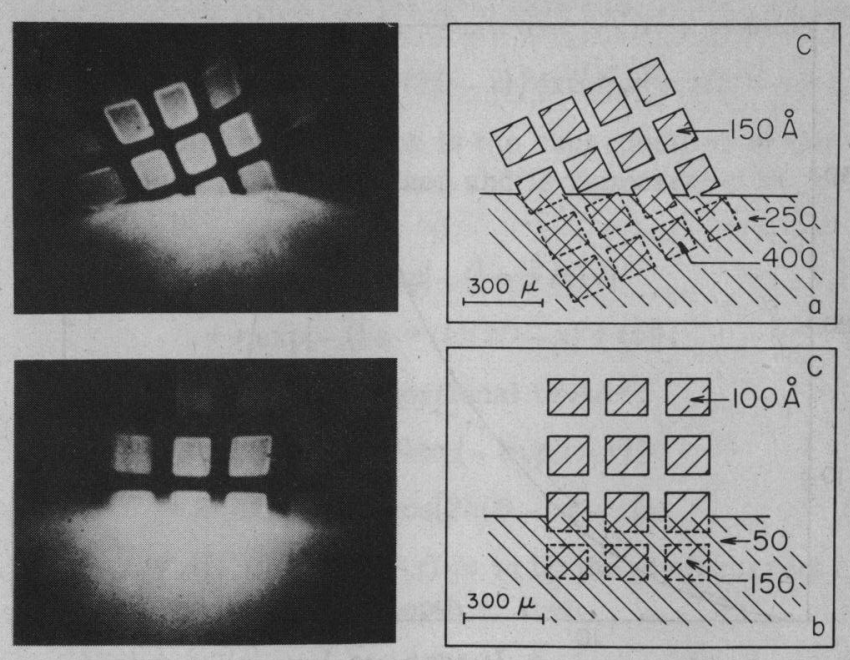

FIG. 6. (a) Phthalocyanine grid pattern, $150 \AA$ thick, partially substrate, (b) Phthalocyanine grid pattern, $100 \AA$ thick, partially covered with an additional $50 \AA$. Carbon-nitrocellulose substrate as in Fig. 5 .

terns have been deposited on a carbon-nitrocellulose We ntre halves of these two microse hesent in the some electrons may originate in the grid pattern and pass through the relatively thin over-layer, the dominant effect in Fig。 4 is clearly reflection from the highly polished stainless-steel substrate. These results in both uniform layers and grid patterns are in agreement with the calculations, and we conclude the reflection model is essentially correct.

\section{ACKNOWLEDGMENTS}

The authors wish to thank George H. Lesch and Dr. W. Engel, Dr. M. Takeo, and Dr. L. Wegmann for Weith $F$. Kongslie for expert by a U.S. Public Health Service Research Grant from the National Cancer Institute.

Y. Harada and H. Inokuchi, Bull. Chem. Soc. Jpn. 39, 144

${ }^{2}$ B. H. Schechtman and W.E. Spicer, J. Mol. Spectrosc. 33 ,

W. Pong and J.A. Smith, J. Appl. Phys. 44, 174 (1973). d.N. Kramer and M. P. Klein, in Electron Spectroscopy,
edited by D.A. Shirley (North-Holland, Amsterdam, 1972),

'K. Siegbahn, C. Nordling, A. Fahlman, R. Nordberg,

K. Hamrin, J. Hedman, G. Johansson, T. Bergmark, S. E. Karlsson, I. Lindgren, and B. Lindberg, in $E S C A$ means of electron spectroscopy (Almqvist and Wiksells, Uppsala, 1967).
${ }^{6}$ O. H. Griffith, G.H. Lesch, G.F. Rempfer, G. B. Birrell, C.A. Burke, D.W. Schlosser, M. H. Mallon, G. B. Lee, Acad. Sci. USA $69,561(1972)$.
${ }^{7}$ R. J. Dam, C.A. Burke, and O. H. Griffith, Biophys. J.

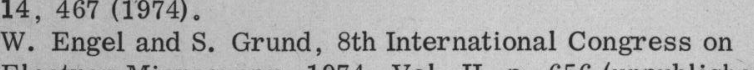
R.J. Dam, G.H. Lesch, D. W. Deamer, and O. H. Griffith 33rd Annual Proceedings of the Electron Microscopy Societ of America, Las vegas, 1975, edited by G.W. Bailey

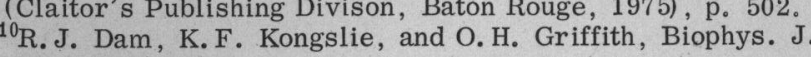

A. Cosslett and V.E. Cosslett, Br. J. Appl. Phys. 8, 374

2C.A. Burke, G. B. Birrell, G. H. Lesch, and O. H ${ }^{13}$ B. H. Schechtman, from Stanford Electronics Laboratories Technical Report No. 5207-2, 1968 (unpublished).
${ }^{14} \mathrm{G}$. Möllenstedt, R. Speidel, and W. Koch, Z Phys ch, Z. Phys. 149 , ${ }^{15}$ L. Wegmann, Mikroskopie 26, 99 (1970). ${ }^{16}$ Carbon films thicker than $260 \AA$ will, of course, reduce the 


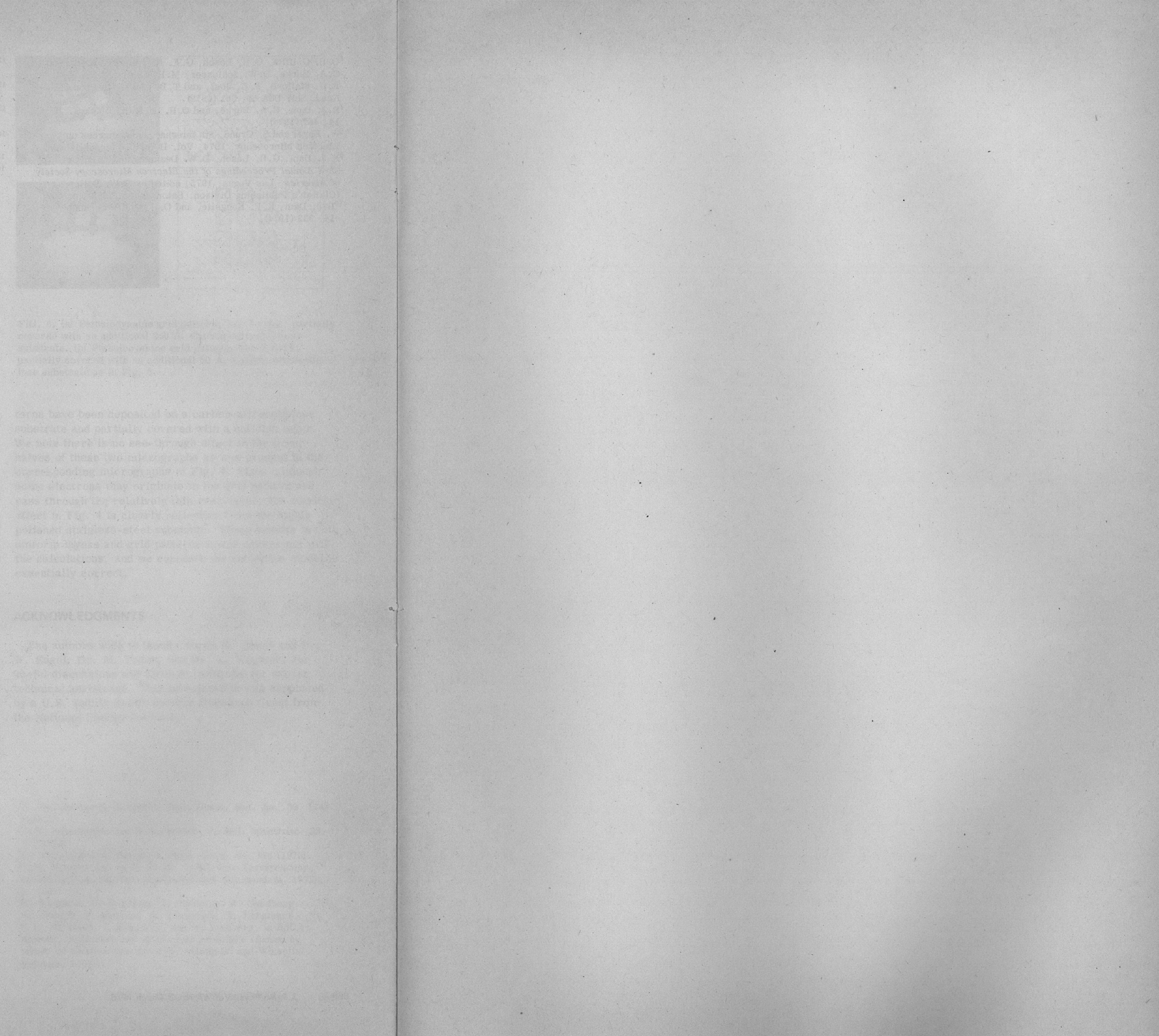


